\title{
Modelling length of stay and patient flows: methodological case studies from the UK neonatal care services
}

\author{
Eren Demir, Reda Lebcir and Shola Adeyemi \\ Hertfordshire Business School Working Paper (2013)
}

The Working Paper Series is intended for rapid dissemination of research results, work-in-progress, and innovative teaching methods, at the pre-publication stage. Comments are welcomed and should be addressed to the individual author(s). It should be noted that papers in this series are often provisional and comments and/or citations should take account of this.

Hertfordshire Business School Working Papers are freely downloadable from https://uhra.herts.ac.uk/dspace/handle/2299/5549 and also from the British Library: www.mbsportal.bl.uk

Copyright and all rights therein are retained by the authors. All persons copying this information are expected to adhere to the terms and conditions invoked by each author's copyright. These works may not be re-posted without the explicit permission of the copyright holders.

The Hertfordshire Business School employs approximately 150 academic staff in a state-of-the-art environment located in Hatfield Business Park. It offers 17 undergraduate degree programmes and 21 postgraduate programmes; there are about 75 research students working at doctoral level. The University of Hertfordshire is the UK's leading business-facing university and an exemplar in the sector. It is one of the region's largest employers with over 2,650 staff and a turnover of almost £233 million. It ranks in the top 4\% of all universities in the world according to the Times Higher Education World Rankings and is also one of the top 100 universities in the world under 50 years old. In the last UK Research Assessment Exercise it was given the highest rank for research quality among the post-1992 universities. 


\title{
Modelling length of stay and patient flows: methodological case studies from the UK neonatal care services
}

\author{
Eren Demir and Reda Lebcir \\ e.demir@herts.ac.uk,m.r.lebcir@herts.ac.uk \\ Department of Marketing \& Enterprise, Business School, \\ University of Hertfordshire, Hertfordshire, UK \\ Shola Adeyemi \\ sholadeyemi2003@yahoo.com \\ Department of Business Information Systems, \\ School of Electronics and Computer Science, \\ University of Westminster, London, UK
}

March 7, 2013

\begin{abstract}
The number of babies needing neonatal care is increasing due mainly to technological and therapeutic advances. These advances have implied a decreasing neonatal mortality rate for low birth weight infants and also a falling incidence of preterm stillbirth. Given the structural changes in the National Health Service in England, coupled with recession and capacity constraints, the neonatal system is facing some serious challenges, such as nurse shortages and the lack of cots, which could inevitably impact neonates length of stay, and the performance of the system as a whole. These constraints have forced neonatal managers to better understand their organisation and operations in order to optimize their systems. As a result, we have developed three unique methodologies based on length of stay modelling, physical patient pathways, and system dynamics modelling. This paper evaluates these techniques applied to neonatal services in London, and showcases their usefulness and implications in practice, particularly focusing on patient flow to determine major drivers of the system, which could reduce inefficiencies, improve patient experience, and reduce cost.
\end{abstract}

Keywords: Patient flow, neonatal care, length of stay, random effects, system dynamics. 


\section{Introduction}

Neonatal services in the United Kingdom (UK) are part of the National Health Service (NHS) and are tasked with providing medical care for babies who develop health complications. These babies are born premature (under 37 weeks), have a low birth weight (under 2,500g) or are born at term with medical problems (BLISS, 2007). These services are under significant pressure as the number of babies requiring such care is increasing. For example, in 2006-07, 62,471 babies, roughly one in ten of all births in the UK, were admitted to neonatal services up from 59,711 in 2005-06 (National Audit Office, 2008). This trend is expected to continue as the number of births in the UK has been increasing in the last few years.

This high demand has put neonatal units under severe strain and there is a growing body of evidence that units are unable to cope with this demand. A recent study reported that over a 6 month period in 2006-2007, neonatal units were shut to new admissions for an average of 24 days. It was found that 1 in 10 units exceeded their capacity for intensive care for more than 50 days during the 6 month period (Asaduzzaman et al, 2009). A recent report found that in 2010, neonatal units in the UK provided a total of 710,631 care-days from which 93,427 care day were for the high cost Intensive Care (NDAU, 2010).

The significant demand for neonatal care coupled with the fact that this care belongs to the high cost category had led to special attention and the scrutiny of the management and performance of neonatal units in the UK. Following a 2008 report by the National Audit Office (National Audit Office, 2008), a neonatal task force was set up by the NHS chief executive with the objective to respond to the report. The task force published a "Neonatal Services toolkit" (Department of Health, 2009), which highlighted, among other things, the importance and urgency of addressing productivity challenges in the UK neonatal services especially in the new public sector where the reality is that more must be done with less.

Neonatal services structure includes two layers of complexity, which affects its ability to cope with demand. First, there are three different levels of care (Intensive, High Dependency and Special) and babies in every level of care are subject to various movements between different intermediate states 
from the entry point (admission state) to the exit points from the system (treatment outcomes states). Second, many babies do not exit the neonatal care system in the level of care in which they were admitted and these babies are subject to transitions between states in different levels of care while in the neonatal system. Combining these two layers of complexity means that neonatal services are a multistate multi care level system in which babies spend some time in some states and care levels and then transit to other ones before they are released from the system.

The time spent in the different care states, known as "Length of Stay (LoS)" is therefore central to the performance of neonatal services as it regulates the pace at which babies flow between these states. This pace determines the number of babies that can be treated in the neonatal units per unit time (one year for example) and the level of utilisation of resources in these services. Furthermore, if we take account of the high cost of neonatal care (Tucker, 2002), then it becomes clear that LoS is a critical driver of both the clinical and economic performance of the neonatal care system.

The impact of LoS on the performance of health care delivery systems is a growing field of interest in health services research and modelling techniques have played a pivotal role in this research (Cooper et al, 2007; Proudlove et al, 2007). This is due to the growing need to understand the complexity of these systems and provide evidence based methodologies to guide clinical and healthcare management policy making.

An overview of past research suggests that four types of methodologies have dominated patient flow and LoS modelling: (i) stochastic modelling, (ii) statistical modelling, (iii) systems dynamics (SD) modelling and (iv) discrete event simulation modelling. Stochastic modelling is a LoS datadriven approach in which the data is allowed to "speak for itself". The objective is to capture the distribution of LoS, which can be explained by loosely defining the flow of neonates. This approach does not impose any preconceptions or clinical/organisational interpretations on it. The statistical approach is about the clinical/organisational based pathways, where we start with the known structure of the real-life system. This model can be used to capture interesting pathways, such as those with the least (or highest) risk of death. Systems Dynamics modelling focuses on the representation of systems whose behaviour over time is driven by feedback processes and exhibits 
high level of dynamic complexity. SD allows the simulation and testing of the behaviour of these systems with the aim of improving our understanding of the inner working of systems and the policies to improve their performance. Discrete-event simulation modelling is a common approach to capture the real life setting of patient pathways (or systems) with the aid of a simulation software (e.g. SIMUL8). It allows the analysis of different scenarios to understand the impact of each decision in complex systems. These techniques are not exhaustive, but nevertheless they are the most widely used approaches to better understand major drivers and the linkages between the operational policies related to LoS and patient flows.

This research aims at advancing our knowledge with regard to the application of modelling techniques to neonatal services. Although the four techniques cited above have been extensively applied in the area of health management, there have been very few applications of stochastic modelling and no applications of system dynamics modelling in neonatal services. This is another important motivator of this research as we would like to assess the usefulness of various modelling techniques in neonatal services and showcase their benefits and implications in practice. By doing so, the aim is to widen the remit of applications of modeling techniques in health care management and assess the pertinence of the insights gained from such exercise.

The paper is organised as follows: Section 2 illustrates the current literature on LoS modelling and patient pathways. Section 3 introduces the methodology for stochastic modelling, where the results are summarised using data from a neonatal unit of a hospital in London. Section 4 describes the statistical framework (physical patient pathways) and summarises the results based on the physical movement of neonates within the neonatal system. Section 5 describes the system dynamics modelling approach and develops a computer simulation model of a neonatal unit in one of London's hospitals. The last section of the paper focuses on the main conclusions of the study and compares and discusses the three approaches in terms of the implications and usefulness in practice. 


\section{Patient flow and length of stay modelling}

Patients flow can be viewed in two perspectives, operational and clinical (Thomas, 1968; Cote, 2000). The common characteristics of both include an entrance, an exit, a path connecting both entrance and exit, and the random nature of the health care elements. This section discusses some of the developments in the area of research in which modelling of patient flow and LOS has been carried out, namely stochastic modelling, statistical modelling and systems dynamic modelling. As our research does not involve the development of a discrete event simulation (DES) model, further literature reviewing related to DES was not carried out. However, readers should note that DES is a popular technique and widely used in the context of examining patient pathways (for further details see (Gunal and Pidd, 2010; Jun et al, 1999)).

\subsection{Stochastic modelling}

Markov models have been used extensively to capture probabilistic laws that govern the dynamics of patients in a healthcare system. For instance, a two-stage continuous-time Markov model that describes the movement of patients through two compartments in geriatric hospitals was developed by Irvine and McClean (1994). Such an approach takes into account different types of patients and their corresponding length of stay. Irvine's (Markov) model (Irvine and McClean, 1994) was further extended to describe the behaviour of patients moving through three stages in a geriatric department (McClean and Millard, 1993). The model estimates the rate of discharge and costs the movements of patients through virtual compartments (or phases), which are loosely defined as acute care, rehabilitation and long-stay care, thus providing a model that can facilitate planning of health and social services for the elderly while taking cost into account. For the first time Cote and Stein (2007) developed a stochastic model (semi-Markov process) to capture the individual patient's experience during a visit to the local family practice clinic, and estimated the transition probabilities between the paths (waiting room, nurse aide station, examining room, lab/x-ray and discharge) visited by the patient.

Phase-type distributions have also been employed to represent the variable nature of LoS. This 
class of distributions describes the time to absorption of a finite Markov chain in continuous time, where there is a single exit (absorbing state) and the stochastic process starts in a transient state (Faddy and McClean, 1999). These models describe duration until an event occurs in terms of a process consisting of a sequence of latent phases (the states of a latent Markov model). However, the generality of the phase-type distributions makes it difficult to estimate all the parameters of the model. To overcome this problem Coxian Phase-type distributions were introduced. Coxian phase-type distributions describe duration until an event in terms of a process which consists of a sequence of latent phases.

\subsection{Statistical modelling}

In the context of LoS and patient flow modelling, few papers have appeared in the application of advanced statistical models, such as Generalized Linear Mixed Model (GLMM) with none known so far applied to capture individual patients' pathway. Using multilevel modelling, Frick et al (1996) compared the influence of individual and organizational variables on patients' LoS in a large psychiatric hospital in Germany and demonstrated the general advantages of mixed effect modelling in the comparisons of hospitals or other health care organizations. A hierarchical Poisson regression model for maternity LoS (Lee et al, 2001) was developed to capture the inherent correlations of patients clustered within hospitals. A finite mixture regression model with random effects and its application to neonatal hospital length of stay has been proposed in Yau et al (2003) leading to the development of the class of finite mixture GLMM where heterogeneity in LoS has been modelled by a two-component mixture model, with one component corresponding to the short stay subpopulation and another component corresponding to the long-stay subpopulation.

Motivated by Cote and Stein (2007) and the aforementioned articles above, Adeyemi et al (2010) developed a multinomial logit model to capture individual patient's pathway in the process of care, where patient frailties (or severity) were modelled as random effects. Two variants of the model were developed, one based on mere patient pathways and the other based on patient characteristics. The approach identified interesting pathways such as those that resulted in high probability of 
death (survival), pathways incurring the least (highest) cost of care or pathways with the least (highest) length of stay. Patient specific discharge probabilities from the healthcare system were also predicted. As an extension, a marginal continuation ratio logit model (Adeyemi et al, 2009b) was further developed to determine the probability of a chronic obstructive pulmonary disease (COPD) patient to be in the high risk group in their next readmission, given patient and regional specific previous readmissions, i.e. capturing individual clinical pathways of patient's leading to multiple readmissions. Here, COPD patients from the North West of England were found to be 16\% more likely to experience multiple readmissions than Yorkshire and The Humber region.

\subsection{Systems dynamics modelling}

System Dynamics (SD) applications in health management have grown significantly in the last decade both in terms of their number and the areas of investigation. This process is driven by the increased awareness that health systems are complex and dynamic in nature, include a dense web of interconnected elements, and are subject to time delays and non linear relationships. Health systems in which patients' flows are core components are a good example of such dynamic complex systems (Dangerfield, 1999; Brailsford et al, 2004; Taylor and Dangerfield, 2005; Lebcir et al, 2010).

Patient flow modelling represents the transition of patients between the different disease and care states and the processes governing these transitions. The primary focus is on the groups of patients sharing the same state rather than on the pathways taken by individual patients especially in contexts where the number of patients is significant. This fits well with SD modelling as in SD the population of patients is divided into large homogenous groups representing the clinical states and patients flows are represented by the rates at which patients move between these states (Brailsford et al, 2004; Atun et al, 2007).

There have been several applications of SD to model patient flows. For instance, Wolstenholme (1999) developed a model to represent patients flows in the UK NHS, which portrayed the movement of patients through the different "routes" in the NHS system with a specific focus on pre-operative and post-operative "intermediate" states. The model showed that reduction in the LoS was a more 
effective policy to reduce the waiting time to admission to hospitals than increasing bed capacity in hospitals. Townshend and Turner (2000) built a model to assess the impact of screening programs on the spread of Chlamydia disease. The model represented the flow of patients between the susceptible, disease, and recovery states. Scenario testing suggested that targeted screening policies would reduce the spread of the disease in a cost-effective manner. In a study of patient flows in an accident and emergency unit in a UK hospital, a model focused on the movement of patients to the unit including patients who are admitted to the hospital wards after their emergency treatment and from GPs (Lane et al, 2000). The model showed that an increase in the hospital bed capacity did not have a significant influence on the waiting time in the A\&E unit. However, even a small increase in accident and emergency demand would increase the LoS in the unit and impact on the patients' total waiting time. A model covering the entire health system of Nottingham (UK), which included patients' flows between the system's units was developed to test policies regarding growth of emergency admissions and reduction of the LoS of elderly patients (Brailsford et al, 2004). Results suggest that a reduction of the LoS made almost no difference to the bed occupancy rate and, hence, had no impact on the system's performance.

The next section describes the Coxian phase-type distribution approach (i.e. stochastic modelling), which is used to capture the LoS distribution of babies in neonatal care. Section 4 illustrates the multinomial random effects modelling approach (statistical model) to capture individual babies pathways (or movements) within neonatal care to explore pathways that may be of an interest to key decision makers. The effects of patient characteristics (LoS, birth weight and gestation age) on these pathways are further investigated. Section 5 develops a system dynamics model to evaluate the impact of introducing new treatment technologies to neonatal care. A qualitative map portraying the inner workings of the neonatal system is produced, followed by the development of a computer simulation model. A number of scenarios were simulated, i.e. examining the impact of reducing LoS. 


\section{Stochastic modelling}

Stochastic networks provide a general and powerful framework to describe LoS data, which can be viewed as generated by some underlying absorbing random process. Phase-type distributions describe the time to absorption (or LoS) of such process. The structure of the stochastic network reflects some prior knowledge on the underlying process. Aalen (1995) reviews the use of phase-type distributions to analyse survival data. Examples of using Coxian distributions in modelling failure time data can be found in Faddy $(1994,1995)$. It should be stressed that the analysis of survival data (or LoS data) using stochastic networks differs in its focus from traditional survival analysis, e.g., using the Cox proportional hazard model. The former concentrates on modelling the random process from which the LoS data is generated, whereas the latter uses LoS data as a vehicle to study the effect of different factors on survival time. Although patient attributes are not usually explicitly modelled in the stochastic network approach, the heterogeneity among patients is reflected in the choice of different paths when progressing through the network.

\subsection{Case study 1: Coxian phase-type distribution}

In the context of the neonatal system, increasing length-of-stay (LoS) in hospital could be related to the severity of the illness being treated. The proposed conceptual model for the movement of neonates in hospital is depicted in Figure 1. Less severe neonates can be discharged (home or death) at an early phase, whereas those associated with more severe illnesses could stay in hospital for a longer period of time before discharge. We construct a continuous-time Markov chain of the flow of neonates in hospital.

Given the model described in Figure 1, the generator matrix is written as

$$
\boldsymbol{\Psi}=\left(\begin{array}{cccccc}
-\left(\lambda_{1}+\mu_{1}\right) & \lambda_{1} & 0 & \ldots & 0 & 0 \\
0 & -\left(\lambda_{2}+\mu_{2}\right) & \lambda_{2} & \cdots & 0 & 0 \\
\vdots & \vdots & \vdots & \vdots & \vdots & \vdots \\
0 & 0 & 0 & \cdots & -\left(\lambda_{k-1}+\mu_{k-1}\right) & \lambda_{k-1} \\
0 & 0 & 0 & \cdots & 0 & -\mu_{k}
\end{array}\right)
$$

where $\lambda_{1}$ to $\lambda_{k-1}$ are the sequential transfer rates between phases, i.e. the rates of the flow of 


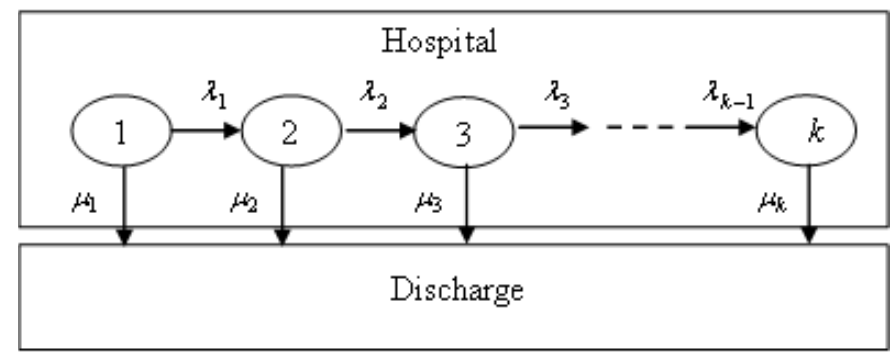

Figure 1: Phases neonates experience in hospital before discharge

neonates in hospital, and $\mu_{1}$ to $\mu_{k}$ are discharge rates from hospital. Thus, the random variable length-of-stay (denoted by $X$ ) has the probability density function (pdf) $f(x)$, given by $f(x)=$ $\boldsymbol{\phi} \exp (\Psi x) \boldsymbol{\alpha}$, where $\boldsymbol{\phi}=\left(\begin{array}{llllll}1 & 0 & 0 & \cdots & 0 & 0\end{array}\right)^{T}$, i.e., the process starts in the first phase, $\boldsymbol{\alpha}=$ $\mathbf{- \Psi 1}=\left(\begin{array}{llll}\mu_{1} & \mu_{2} & \cdots & \mu_{k}\end{array}\right)^{T}$ and $\mathbf{1}$ is a column vector of 1's. The resulting pdf could also be expressed as a mixture of generalized Erlang distributions (Ross, 2003) with probability $p_{1}=$ $\mu_{1} /\left(\lambda_{1}+\mu_{1}\right)$ absorption (or discharge) is from phase 1 , or with probability $p_{2}=\lambda_{1} /\left(\lambda_{1}+\mu_{1}\right)$. $\mu_{2} /\left(\lambda_{2}+\mu_{2}\right)$ absorbtion is from phase 2, and so on. Given data consisting of observed length of stay, $x_{1}, x_{2}, x_{3}, \ldots, x_{s}$, the $2 n-1$ parameters describing the LoS distribution is determined by maximizing the log-likelihood function using a statistical computing language $\mathrm{R}(\mathrm{R}, 2005)$ (see Faddy and McClean (1999) for further details).

\subsection{Neonatal Dataset}

To perform this analysis, one-year data (2006) were collected from the North Central London Perinatal Network (NCLPN). The database system known as Standardised Electronic Neonatal Database (SEND) was established in 2006 to collect information from the network. The SEND system allows the evaluation of the activity across the network and sharing of clinical information as neonates transfer between units. The NCLPN is made up of 6 hospitals including University College London Hospital (UCLH) which is the perinatal centre (level 3), two level 2 hospitals (Barnet and Whittington hospitals) and two level 1 hospitals (Royal Free Hampstead and Chase Farm hospitals). 
There were 1002 neonates admitted to UCLH in 2006, of which 993 were observed cases (9 missing), where 830 booked for delivery at the neonatal care of UCLH and 172 from other hospitals, either inside or outside the NCLPN. The majority of babies are discharged home (about 71\%); only few die (about 1.9\%); the others are referred to wards within the hospital (about $7.2 \%$ ) or to other hospitals (about 18.6\%) for cardiac care, critical care, special care or surgery. Only a very small number of records have missing discharge destinations.

\subsection{The application of Coxian phase-type distribution}

A sequential procedure was adopted (as in Faddy and McClean (1999)) whereby increasing numbers of phases $k$ were tried, starting with $k=1$ (corresponding to the exponential distribution), until a fit was obtained that satisfactorily described the main features of the observed data. The initial parameters for each model $(n=1, . ., k)$ were determined by fitting a mixture of exponential distributions. The results of fitting phase-type distributions to the neonatal dataset is summarized in Table 1.

Table 1: Model selection
\begin{tabular}{|c|c|}
\hline Phases & log-likelihood value \\
\hline$k=1$ & -3475.577 \\
$k=2$ & -3321.177 \\
$k=3$ & -3296.036 \\
$k=4$ & -3296.846 \\
\hline
\end{tabular}

Judging by the log-likelihood values, $k=3$ phases were found to provide "best" fit of the data. Furthermore, visual inspection of the empirical histogram and fitted pdf curve in Figure 2, show a close agreement and suggest that the selected model is able to capture the overall patterns of LoS. The maximum likelihood estimates were (with standard errors in brackets): $\hat{\lambda}_{1}=0.26717(0.04129), \hat{\lambda}_{2}=$ $0.09094(0.01842), \hat{\mu}_{1}=0.07842(0.01098), \hat{\mu}_{2}=0.25496(0.02991)$ and $\hat{\mu}_{3}=0.03046(0.00292)$.

The phases can be loosely defined as "short stay", "medium stay" and "long stay". The probability of discharge from these phases can be estimated, thus the probability of discharge from short, medium, and long stay group is estimated to be $0.23,0.57$, and 0.20 , respectively (with a mean length of stay of 3,6 , and 39 days, respectively). 
A danger of this loose definition is that readers may associate short, medium and long stayers with special care (SC), high dependency care (HDC) and intensive care (IC) units, respectively. Note that neonates could only physically move from IC to HDC to SC (not vice versa) and then be discharged from hospital, whereas here, increasing length of stay (from phase 1 to phase 3 ) is associated with severity and not with physical movement of neonates. Nevertheless, the phases to some extent can be associated with these units, i.e. a special care unit neonate would be expected to stay in care shorter than their HDC or IC counterparts. The advantage of this approach is the simplicity in capturing the distribution of LoS solely based on a single variable, which could easily be derived from most routinely collected administrative datasets. Furthermore, it provides a high level overview of neonatal care services with respect to LoS, e.g. probability of discharges and average LoS within each phase. In the commissioners, clinicians (and/or neonatal managers) perspective this approach could enable them to gain a better insight into the main driver that regulates the pace at which babies are admitted into care and the level of utilisaton of resources.

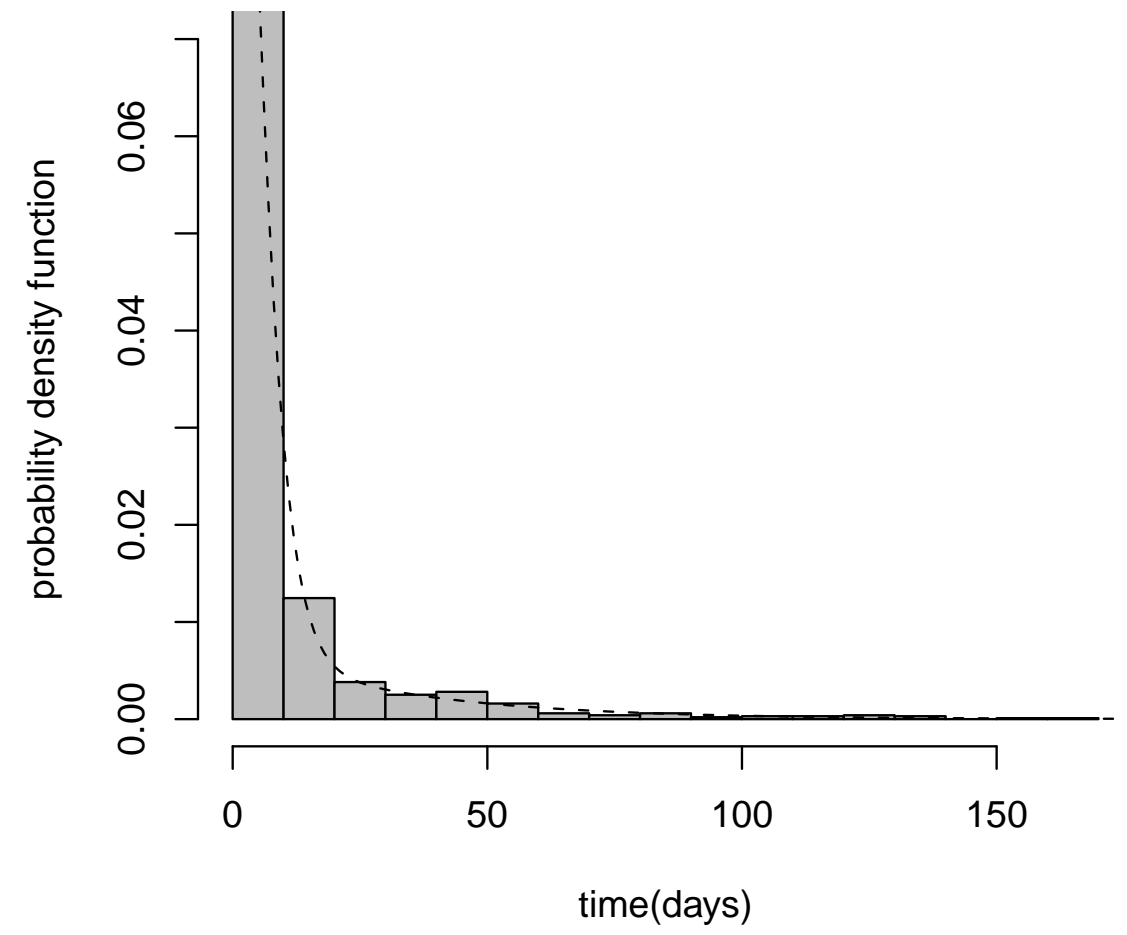

Figure 2: Observed (histogram) and fitted (dotted line) probability density function 


\section{Physical Patient Pathways}

Adeyemi et al. (Adeyemi et al, 2007, 2009a, 2010, 2011) developed series of random effects modelling frameworks to capture physical patient flow paths through the healthcare system. The Coxian phase-type distribution approach presented above assumed virtual or latent phases (i.e. hypothetical system), whereas this approach deals with the real system, typically identifying the states within the system that a patient has passed through. This approach provides a convenient way to capture association and unobserved heterogeneity, such as identifying pathways that result in high probability of death (survival) or pathways incurring the least (highest) cost of care or pathways with the least (highest) length of stay. Patient-specific discharge probabilities from the healthcare system could also be predicted. This is very important from a management perspective since variability within the system is what makes planning and management difficult. We firstly introduce the modelling framework here and apply this method to the neonatal dataset provided by a hospital in central London.

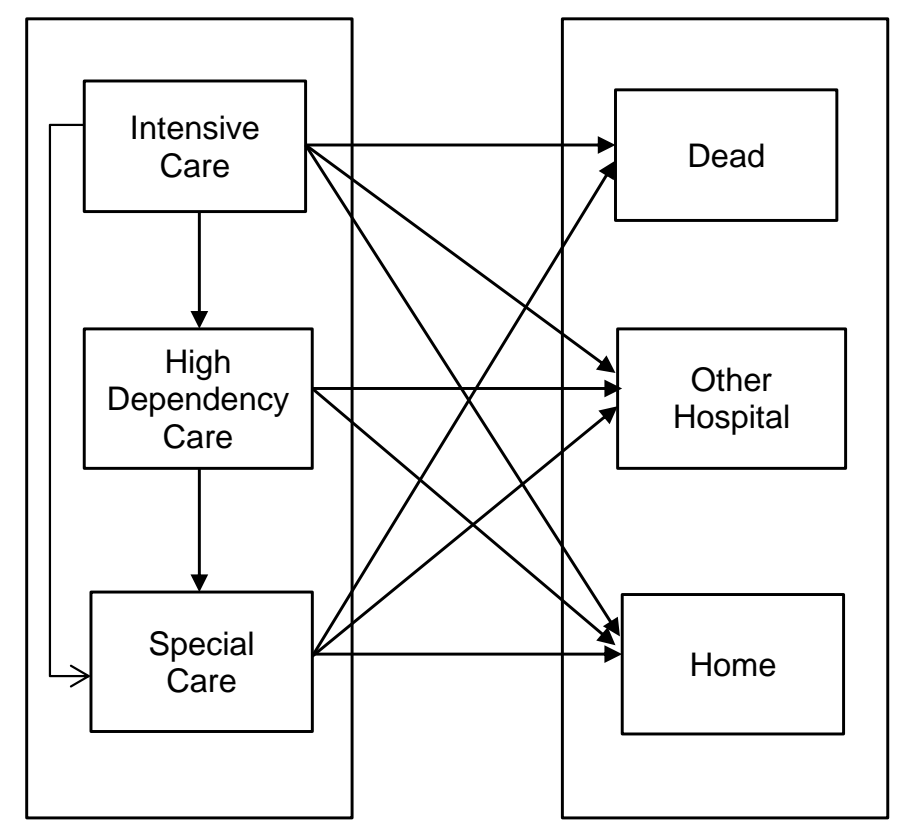

Figure 3: Pattern of Flow for Neonates between Units of Care 


\subsection{Case study 2: Multinomial random effects modelling}

Figure 3 illustrates the physical movements of neonates, which is the core piece of information required as well as transfers and discharge destinations. Discharge from each unit could be by death, transfer to other hospitals $(\mathrm{OH})$ or home. From the data provided by UCLH, we notice that all movements are forward, hence the models proposed here do not capture backward flow of babies (e.g. special care to HDC and HDC to intensive care). The three levels of care are assumed to be some transient states that the newborn babies experience until they are discharged. So, the probability of passing through any of the paths is modelled as a random effects multinomial logit model (see Adeyemi et al (2010) for details).

The probability of patient $p$ passing through any of the paths is a function of the patient specific frailty, such that the movement of babies through the system is being driven by these random effects (frailties). The concept of frailty of a newborn is modelled as a random effect. The key idea is that an individual baby possesses different frailties, and those babies who are most frail will die earlier while others continue to flow in the system until they are discharged. Frailty is further modelled by a set of clinical covariates, such as length of stay of babies, their gestation age and birth weight.

In this application, we have fitted two models for this purpose. The first is a model (model I) without any covariates in which the pathways probabilities are modelled as functions of patient experience during the process of care and patients frailties. The second models pathways probabilities as functions of patient characteristics (birth weight, total length of stay and gestation age) and frailties.

\subsection{Results}

Table 2 and 4 provide parameter estimates and standard errors of our neonatal data set for the described models. Model I depicts satisfactorily the activities in the neonatal system described in the previous section. The model shows significant activities $(p<0.05)$ in almost all pathways except HDC - OH, IC - HDC - OH, HDC - SC - OH, IC - HDC - SC - OH, HDC - Home and IC - HDC - SC - Home, where there are very few or no movements. These are consistent with the activities 


\begin{tabular}{|l|l|l|}
\hline Pathway & Estimate & Standard Error \\
\hline \hline IC - OH & 2.4400 & $0.5018^{\star}$ \\
HDC - OH & 0.8612 & 0.7852 \\
SC - OH & 0.8178 & $0.1020^{\star}$ \\
IC - HDC - OH & 0.2398 & 0.4984 \\
IC - SC - OH & -1.3458 & $0.4294^{\star}$ \\
HDC - SC - OH & -0.1295 & 0.6996 \\
IC - HDC - SC - OH & 0.8139 & 1.3684 \\
IC - Home & 3.3311 & $0.4718^{\star}$ \\
HDC - Home & 3.1274 & 1.2011 \\
SC - Home & 1.8846 & $0.0808^{\star}$ \\
IC - SC - Home & -2.1498 & $0.3419^{\star}$ \\
HDC - SC - Home & -2.8357 & $1.2071^{\star}$ \\
IC - HDC - SC - Home & 1.8630 & 1.2839 \\
$\sigma_{\theta_{p}}^{2}$ & 0.0004 & 0.0037 \\
\hline \hline Fit Statistics & & \\
-2 Log Likelihood & 3331.1 & \\
AIC (smaller is better) & 3365.1 & \\
BIC (smaller is better) & 3448.4 & \\
\hline
\end{tabular}

Table 2: Parameter Estimates and Standard Errors for Model I. $\star$ indicates significance at $p<0.05$.

depicted in Figure 3. However, other pathways have significant effects. Since the interpretations of these significant effects are functions of patient specific frailties, we have presented in the figure below (Figure 4) some plots which show the probabilities of passing through each of the pathways as a function of the frailties. Frailties, $\theta_{p}$ are assumed to follow a multivariate normal distribution. The model estimates of these frailties are incorporated to obtain the multinomial probabilities used in constructing the plots in Figure 4. Transitions between the units are assumed to be driven by these frailties. For instance, discharge home is more likely to occur in babies with increasing LoS (see Figure 4). Transfer from IC to SC is most probable for babies who have being in care (or LoS) between 34 and 40 days, whereas transfer from IC to HDC or HDC to SC are most probable at around a LoS of 31 days. LoS of 31 serves as the threshold below which the probability of discharge by death is most probable while babies who survive this first 31 days have a high chance of being discharged home. This shows that the most critical units of a neonatal system is the IC SC units. One observes from Table 3 that the average LoS for babies moving from ICU-HDU-SCU is around 32 days (similarly ICU-HDU is 33 days), which is around the threshold of 31 days, revealing the 
fact that beyond this threshold the probability for a baby to be discharged alive from hospital is greater. Note that our finding is not conclusive and clearly there is a scope for further research here to investigate the reasons for this apparent threshold.

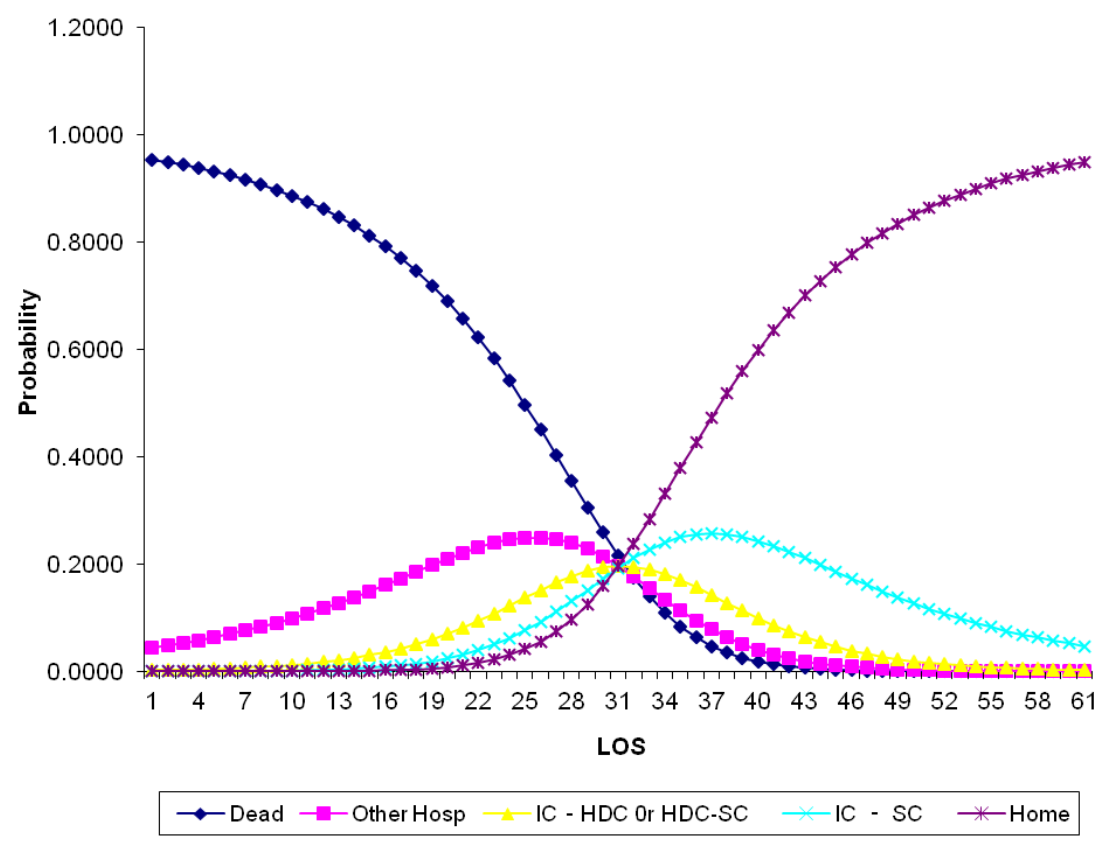

Figure 4: LoS versus flow of babies

\begin{tabular}{|l|c|c|c|}
\hline Pathway & Mean & Median & Standard Dev \\
\hline Dead & 9 & 3 & 15.9 \\
Other Hospitals & 14 & 7 & 18.7 \\
Home & 10 & 6 & 15.0 \\
ICU - HDU - SCU & 32 & 24 & 24.2 \\
HDU - SCU & 16 & 13 & 12.5 \\
ICU - SCU & 27 & 13 & 29.7 \\
ICU - HDU & 33 & 24 & 24.2 \\
SCU Only & 6 & 5 & 4.7 \\
HDU Only & 1 & 1 & - \\
ICU Only & 17 & 7 & 20.4 \\
\hline
\end{tabular}

Table 3: LoS attributes based on the flow of babies

Model II provides significant $(p<0.05)$ effects of all covariates except that birth weight does not significantly affect the pathways ending by a discharge to other hospitals and home (Table 4). Pathways ending with discharge home are highly influenced by gestation age, birth weight and total 


\begin{tabular}{|l|l|l|}
\hline Pathway & Estimate & Standard Error \\
\hline \hline Constant: OH & 2.1116 & $0.7492^{\star}$ \\
Constant: Home & 2.6668 & $0.6063^{\star}$ \\
LOS: OH & 0.6584 & $0.0040^{\star}$ \\
LOS: Home & 0.6679 & $0.0028^{\star}$ \\
Gestation: OH & 4.2901 & $0.0257^{\star}$ \\
Gestation: Home & 4.2813 & $0.0205^{\star}$ \\
Birth weight: OH & 0.1792 & 0.1134 \\
Birth weight - Home & 0.4277 & 0.0881 \\
$\sigma_{\theta_{p}}^{2}$ & 0.9985 & $0.0017^{\star}$ \\
\hline \hline Fit Statistics & & \\
-2 Log Likelihood & 2949.8 & \\
AIC (smaller is better) & 2983.8 & \\
BIC (smaller is better) & 3066.5 & \\
\hline
\end{tabular}

Table 4: Parameter Estimates and Standard Errors for Model II. $\star$ indicates significance at $p<0.05$.

length of stay. The longer the total length of stay the more likely of passing through the pathways ending with a discharge home and this also applies to birth weight and gestation age. Babies with high gestation age and birth weight are likely to pass through pathways ending with a discharge home. The attributable variance at patient level for Model I is insignificant and very significant for Model II (without pathways information) in terms of rejecting the null hypothesis that the patient population standard deviation equals zero. This means that in Model I the null hypothesis of no frailty cannot be rejected, while it can be rejected in Model II. This demonstrates that modelling individual patient pathways captures a substantial part of the variability in the system induced by frailties or individual differences, while this variability is not captured with the model based only on patient characteristics.

\section{Dynamic Modelling of Patient flows}

Patient flow modeling has been a central theme in health care system dynamics modeling. In the context of neonatal care, babies are grouped in different states upon their admission to the unit and then flow between these states as they get treatment in the unit (Department of Health, 2009). In the next section, a System Dynamics simulation model of a neonatal unit in one of the hospitals 
in London, UK focusing on the impact of LoS on patient flows and the performance of the unit is presented.

\subsection{Case study 3: System Dynamics Modelling}

This case study took place in a neonatal unit located in one of the hospitals in North West London. The unit plays a pivotal role in neonatal care in the area as it includes facilities and expertise which enable it to admit and treat babies in all three levels of care (special, high dependency, and intensive care). Furthermore, the unit receives babies from both the hospital where it is located and from all the neonatal units in the area.

The aim of the research was to evaluate the impact of introducing new treatment technologies in the unit. The rationale is that these new technologies would have a positive effect on LoS, which, in turn should improve the performance of the unit in terms of the overall number of babies admitted and treated per year. However, prior to the introduction of these new technologies, the unit management wanted to quantify their likely impact at the unit level so that the implementation process is "evidence-based". This was extremely important as there were doubt on whether the reduction of LoS at the individual level (micro level) will translate into an improvement at the unit level (macro level) given the complex interactions between the elements of the unit.

The modelling process included two main phases. First, a qualitative map portraying the inner working of the unit was developed and included neonatal clinical and medical information and regulations, management procedures, and the decision making process. This was followed by a simulation model building phase where the qualitative map was then translated into a computer based simulation model (iThink version 8.0) representing the functioning of the unit. Several rounds of information gathering and model improvement took place before the management and clinical staff of the unit became satisfied that the computer simulation model is a good representation of the unit structure and processes.

The core component of the simulation model portrays the pathways and flows of patients (babies) within the unit. In this context, babies entering the unit for treatment are allocated to one of the 
three possible care states IC, HDC, or SC depending on their clinical condition. This is followed by a treatment phase from which the outcomes are (i) discharge, (ii) transfer to another unit (hospital), (iii) death, and (iv) transfer to other wards in the hospital where the unit is located. The previous outcomes are valid for babies in all the three states of IC, HDC, and SC. Additionally, there are state dependent outcomes of the treatment phase. Babies in the IC state can move to the SC or HDC state if their situation improves after the treatment phase. Babies in the HDC state can move to the SC state if their situation improves or the IC state if their situation worsens following the treatment phase. Additional possible treatment outcome for babies in the SC state include transitions to the IC or HDC state if the babies' clinical condition worsens. As an illustration, the states and transitions associated with the SC state are presented in Figure 5. The flows of babies in the unit are governed by the different transition rates, namely the admission rate and the treatment rate. The admission rate is determined by the daily admission demand (number of babies requiring admission to the unit per day) and the unit admission policies, which stipulate that admission is restricted only to babies from the hospital where the unit is located if the unit is near saturation.

$$
\begin{aligned}
A D R_{t} & =D E M_{t} \times E C A D R_{t} \\
E C A D R_{t} & =f\left(\frac{\text { OCCOTS }}{\text { TOTCOTS }}\right)
\end{aligned}
$$

where

$A D R_{t}:$ Admission Rate

$D E M_{t}$ : Daily admission demand

$E C A D R$ : Effect of cot occupancy on admission rate

$O C C O T S_{t}:$ Occupied cots

TOTCOTS : Total cots

The treatment rate (the number of babies treated daily), is determined by the rate related to the LoS, which reflects the medical technologies, regulations, and practices in the unit and the rate 


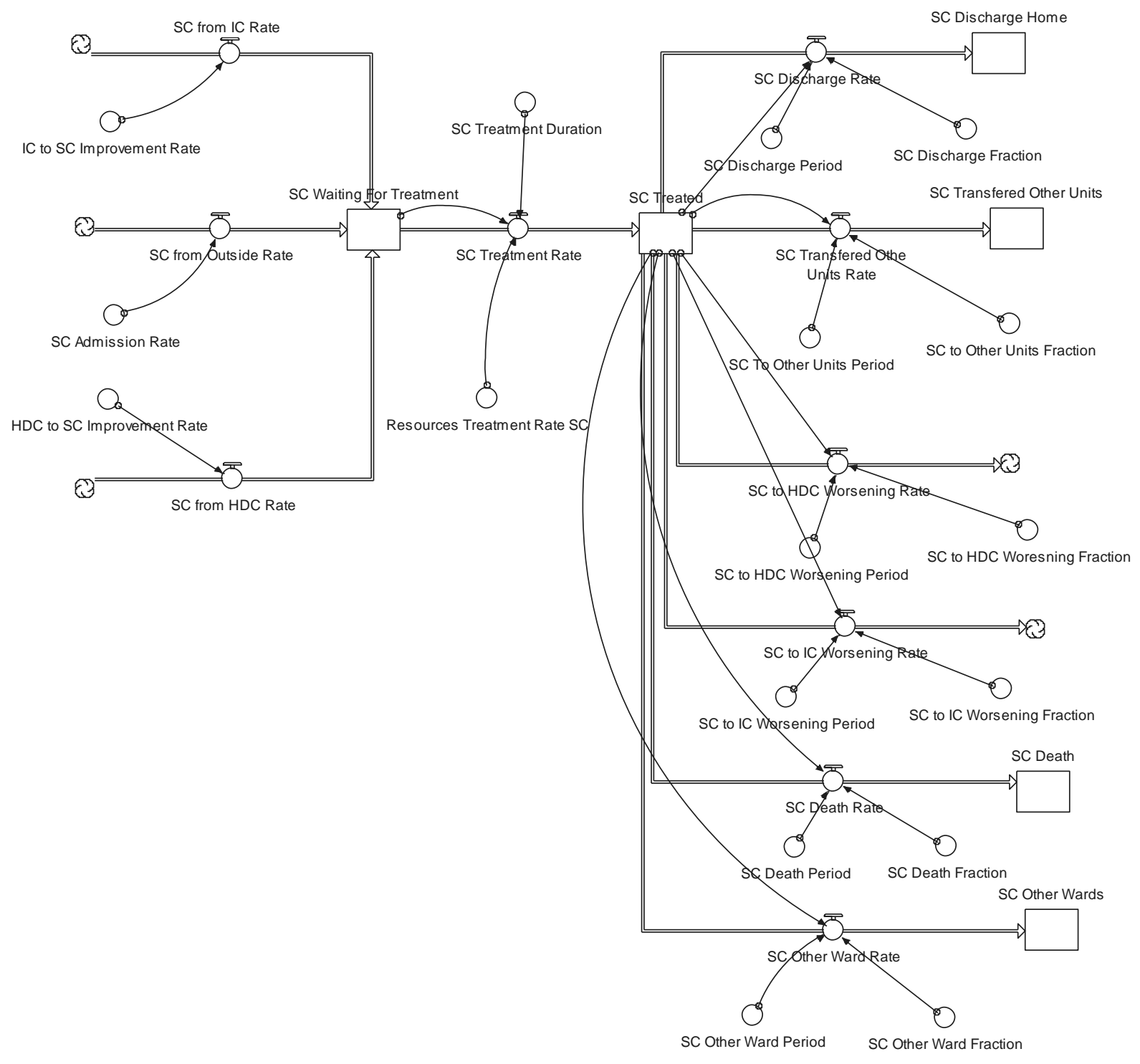

Figure 5: States and transitions associated with the special Care state

related to the availability of resources required to treat babies.

$$
T R M R T_{t . j}=\operatorname{Min}\left(L O S R T_{t . j} ; R E S R T_{t . j}\right), \quad j \in\{S C, H D C, I C\}
$$

where

$T R M R T_{t . j}:$ Treatment Rate

$L O S R T_{t . j}:$ Treatment rate allowed by LoS 
$R E S R T_{t . j}:$ Treatment rate allowed by resources

Equation 4 is important as it represents and explicitly distinguishes between the effect of LoS as determined by the clinical needs and regulations (LOSRT) and the effect of the organisational policies in terms of the availability of resources (RESRT) on the treatment rate of babies.

The treatment rate determined by LoS depends on the number of babies requesting treatment (treatment demand) and LoS

$$
\operatorname{LOSRT}_{t . j}=\frac{B A B_{t . j}}{L O S_{j}}, \quad j \in\{S C, H D C, I C\}
$$

where

$B A B_{t . j}:$ Number of babies requesting treatment

$L O S_{j}$ : Length of stay

The treatment rate determined by the availability of resources depends on the mix of resources required to treat babies, which are (i) cots, (ii) nurses, and (iii) doctors. Given that the treatment of a baby requires the availability of these three resources, the rate equation has the following generic form

$$
R E S R T_{t . j}=\operatorname{Min}\left(C O T_{t . j} ; N U R S_{t . j}, D O C_{t . j}\right), \quad j \in\{S C, H D C, I C\}
$$

where

$R E S R T_{t . j}:$ Treatment rate allowed by resources

$C O T_{t . j}$ : Treatment rate determined by the availability of cots

$N U R S_{t . j}$ : Treatment rate determined by the availability of nurses

$D O C_{t . j}$ : Treatment rate determined by the availability of doctors

The cots treatment rate depends on the number of babies requesting treatment, the number of 
cots in the unit (by category) and the cot-babies treatment ratio (determined by the regulation). The doctors and nurses treatment rates depends on the number of babies requesting treatment, the number of doctors and nurses (by category), the number of babies who can be treated by the different categories of doctors and nurses, and the fraction of labour allocated to treatment activities. The latter factor takes account of issues such as leave, absences due to sickness, and time allocated by doctors and nurses to non-clinical duties (administrative and managerial duties).

The model was validated through a set of qualitative, quantitative, and behavioural reproduction tests (Sterman, 2000; Barlas, 1996). The qualitative tests included verification, by the unit clinical and managerial staff, of the relevance of the variables in the model and the accuracy of all the causeeffects relationships. The quantitative tests included verification of the model equations dimensional consistency, the real world meaning of all variables in the simulation model, and the accuracy of the data in the model. This was done with the involvement of the unit data manager, clinical, and managerial staff. The behavioural reproduction tests included comparison of the simulation results over a period of one year to the real world observations over the same year. The success of the model to replicate real world observations regarding the cumulative number of babies associated with the treatment outcomes (the main performance criteria for the unit management) was significantly high as shown in Table 5 .

\begin{tabular}{|l|c|c|}
\hline Outcome Variable & Real World & Simulation \\
\hline Babies transferred to other units IC & 117 & 108 \\
\hline Babies transferred to other units HDC & 10 & 10 \\
\hline Babies transferred to other units SC & 84 & 70 \\
\hline Babies discharged home IC & 9 & 7 \\
\hline Babies discharged home HDC & 5 & 4 \\
\hline Babies discharged home SC & 196 & 202 \\
\hline Babies transferred same hospital IC & 14 & 12 \\
\hline Babies transferred same hospital HDC & 1 & 1 \\
\hline Babies transferred same hospital SC & 122 & 123 \\
\hline Babies deaths IC & 37 & 31 \\
\hline Babies deaths HDC & 0 & 0 \\
\hline Babies deaths SC & 0 & 0 \\
\hline
\end{tabular}

Table 5: Validation results from the simulation model and real world observations 
Given the aims of the research, the scenarios simulated in this study focused on the impact of reducing LoS on the unit performance. In this context, scenarios included decreasing LoS by 1 day and 3 days for each level of care individually and then for the three levels of care simultaneously. These scenarios were informed by the new treatment technologies considered for introduction in the unit.

The model was run over a period of one year. The performance indicators of interest to the unit management team were related to the cumulative number of babies leaving the unit after treatment and the number of babies refused access to the unit. Therefore, simulation results were collected with regard to the variables "cumulative number of babies discharged home (CBDCH)", "cumulative number of babies transferred to other units (CBTOUT)", "cumulative number of babies transferred in the same hospital (CBTSH)", and "cumulative number of babies refused entry to the unit (CBREU)". The results are presented in Table 6. It is important to notice that these performance indicators do not reflect issues related to the quality of life or the impact of LoS on the quality of care. This is because the clinical and management team in the unit indicated that LoS did not have a direct impact on quality of care as the clinical and treatment procedures were highly standardized in neonatal care and that these procedures were robustly followed in the unit.

\begin{tabular}{|l|c|c|c|c|}
\hline Scenario & CBDCH & CBTOUT & CBTSH & CBREU \\
\hline Baseline (current situation) & 230 & 187 & 136 & 18 \\
\hline Scenario 1: Reduction LoS 1 day SC & 234 & 182 & 138 & 18 \\
\hline Scenario 2: Reduction LoS 3 days SC & 235 & 182 & 139 & 17 \\
\hline Scenario 3: Reduction LoS 1 day HDC & 234 & 182 & 138 & 18 \\
\hline Scenario 4: Reduction LoS 3 days HDC & 233 & 181 & 138 & 20 \\
\hline Scenario 5: Reduction LoS 1 day IC & 233 & 181 & 138 & 21 \\
\hline Scenario 6: Reduction LoS 3 days IC & 234 & 181 & 138 & 20 \\
\hline Scenario 7: Reduction LoS 1 day SC, HDC, IC & 236 & 182 & 139 & 16 \\
\hline Scenario 8: Reduction LoS 3 days SC, HDC, IC & 240 & 185 & 139 & 12 \\
\hline
\end{tabular}

Table 6: Simulation results regarding reduction of LoS

These results show that the reduction of LoS for the three categories of babies (SC, IC, HDC) increases the cumulative number of babies discharged home, transferred to other units, and transferred in the same hospital. However, it is important to notice that this performance improvement 
is similar whether LoS is reduced by 1 day and 3 days for the same state. For example, reducing LoS by 1 and 3 days for the IC state leads to a CBDCH of 233 and 234 babies respectively, a CBTOUT of 181 babies, and a CBTSH of 138 babies. This means that other forces are at play in the system which nullifies the effects of reducing LoS from 1 day to 3 days. These unexpected results reflect the effect of "policy resistance", which is common in dynamically complex systems such as neonatal units.

Reducing LoS simultaneously for the 3 states improves performance especially with respect to $\mathrm{CBDCH}$ and CBREU, which are the most important indicators for the unit's management. However, it is interesting to observe that CBTSH remains at 139 whether LoS is reduced by 1 or 3 days. This is another consequence of policy resistance and its effects on the behaviour of systems.

The results indicated that CBREU increases when LoS is decreased by 3 days for the IC state and by 1 and 3 days for the HDC state (see Figure 6). These results were surprising to the unit management team as one would expect CBREU to decrease (or at least remain unchanged) if LoS is reduced. This is a vivid example of the counter-intuitive behaviour of systems involving high levels of dynamic complexity and how such systems react unexpectedly to policies aiming at improving their performance (Sterman, 2000).

The unexpected results described above can be explained by the Causal Loop Diagram (CLD) presented in Figure 7. This CLD represents babies in the HDC state (similar CLDs represents the IC and SC states but these are not shown here due to lack of space). From the CLD, we can see that a reduction in the LoS leads to an increase in the HDC treatment rate causing a faster movement of babies through balancing loop B2. This would have improved the performance of the unit if it was not for its side effects on reinforcing loops R1 and R2, which represents the process by which some of the HDC babies move to the SC state, get treated and then worsen again and go back to the HDC state (Loop R1) and the process by which some of the HDC babies move to the IC state, get treated and then improve and go back to the HDC state (Loop R2). As balancing loop B2 gets stronger, it triggers a reaction from reinforcing loops $\mathrm{R} 1$ and $\mathrm{R} 2$, which become dominant in the structure and overcome the extra power given to balancing loop B2 (through the reduction in LoS). 


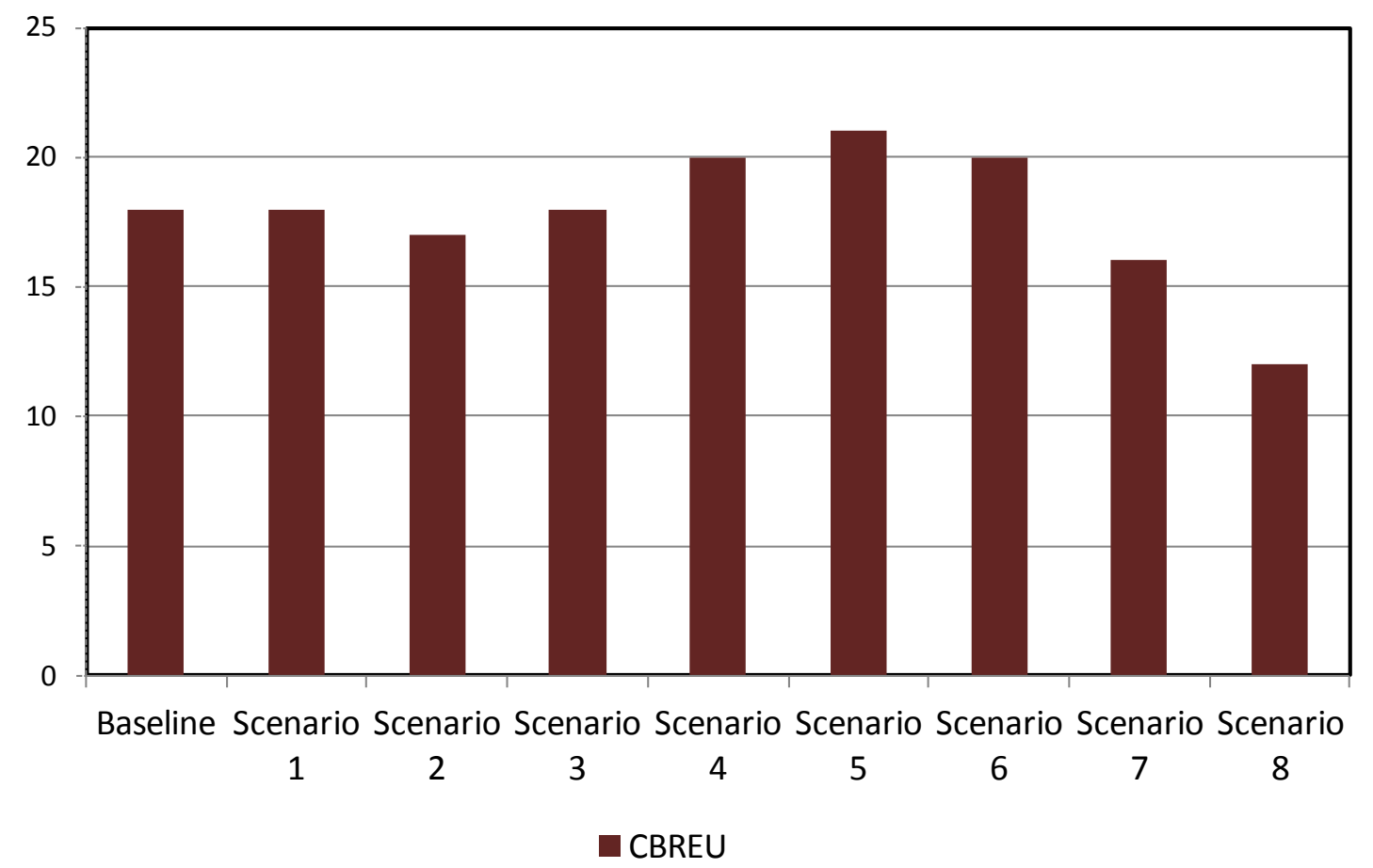

Figure 6: CBREU for the baseline and simulated scenarios

Therefore, a higher number of babies remain within the unit leading to a reduction in the babies leaving the unit and blocking babies from entering it, hence increasing the number of babies refused entry. This is a vivid example of the interaction and power shifts between feedback loops, which is the main driver of counterintuitive behaviour of dynamically complex systems (Sterman, 2000).

This finding illustrates how valuable SD modelling is in improving our understanding of the behaviour of complex systems and informing the process of decision making and policy design.

The managerial implication of these results is that reducing LoS will not lead to substantial improvement in the performance of the unit (in fact it may lead to the contrary under some scenarios). This means that a further analysis of the structure of the system will have to be conducted to determine the root causes of its behaviour and determine the policies which could have a significant influence on the performance of neonatal units. 


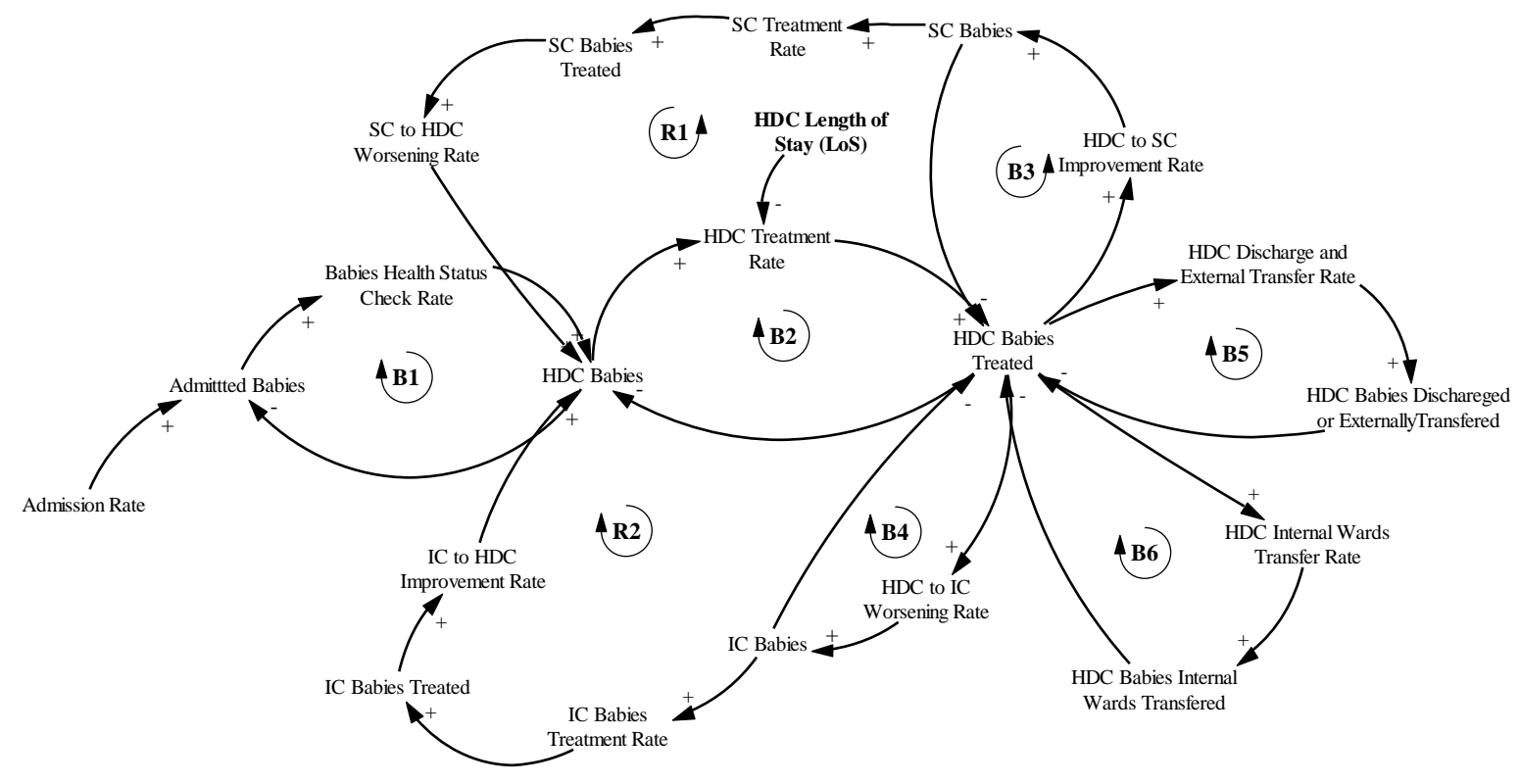

Figure 7: Causal Loop Diagram for the HDC state

\section{Discussion and Conclusion}

The current research addresses a top of the agenda issue in health management as it focuses on the policies related to LoS and how they impact on the performance of health systems. The importance of the research can be appreciated in the current context of increasing demand on health services provision at the time when we are moving to the new reality of tighter public finances and the resulting pressure to improve the effectiveness and efficiency of health care provision and delivery.

The importance of studying LoS is twofold: (i) First, it has a direct impact on the patients flows in health systems, therefore it determines the number of patients going through the system per unit time and (ii) LoS is a reliable proxy measure for the consumption of resources in health systems, which relate directly to the economic performance of these systems.

The methodologies applied in this research generated findings which can be very useful in guiding decision making. The three-phase distribution provided us with valuable information regarding the flow of neonates through the system. We calculated LoS statistics such as the average LoS of a baby in each phase and various probabilities such as the probability that a baby moves from one phase in 
the system to another phase. For instance, suppose there are 20 babies in neonatal care, we could estimate the number of babies falling into each phase, i.e., $23 \%$ is expected to be short stayers (4 babies) with an average length of stay of 3 days; $57 \%$ medium stayers, approximately 11 babies with an average length of stay of 6 days, and the remaining are long stayers. In addition, the estimated parameters can be substituted into the phase-type model for representing the continuous survival times of patients in hospital. This information is important for effective planning of resources and the prediction of future patient $\operatorname{LoS}$ in hospitals, thus it enables achievement of better services provision and clinical outcomes.

The concept of modelling individual neonates' experience during the process of care gave us valuable information about the inner working of the system, (e.g. transfer from IC to SC is most probable for babies who have been in care (or LoS) between 34 and 40 days), a useful information for better planning of the care process. According to BLISS (2007), $78 \%$ of all neonatal units in the UK had to close to new admissions at least once in the last six months. Rejecting premature babies could create risk and the expensive transfer of sick babies to other hospitals. Furthermore, cots in neonatal units are very expensive to maintain and the management wants to optimise their utilization. Therefore, this model could help predict the outcome of a baby (moving to another unit of care, home, other hospital or death) given his/her birth weight, gestation age and the unit of care they are admitted to, hence improving the efficiency and effectiveness of resources allocation policies.

The System Dynamics simulation model represented the pathways in the neonatal system and the processes governing the flow of patients on these pathways. In determining the patients' flows, the model includes different variables to represent the LoS determined by the clinical rules and regulations and the LoS determined by organisational constraints such as resources availability. As such, the model allows the evaluation of a range of policies impacting LoS, and covering both the clinical aspects (e.g. the introduction of a new treatment procedure) and the managerial aspects (e.g. increasing the number of cots) and their impact on the number of babies treated in the unit and their associated outcomes. Furthermore, SD modelling allows a better understanding of the linkages between the structure of the neonatal unit and its behaviour (clinical and managerial outcomes) and how policies lead to the possible results. Beyond the usefulness of the model to guide policy 
making, this improved understanding is important on its own right.

The research has some methodological and contextual limitations. The three phase distribution is not incorporated within a software package, and requires the development of a fitting algorithm by using a general method of maximizing the log-likelihood function. The physical patient pathways modelling has two main shortcomings towards the implementation of this approach in practice: 1) data related to tracking individual patient pathways and outcomes longitudinally over the full care cycle may not be available, if it is, it may sometimes be cumbersome to extract such data as this may involve consulting patients' case notes especially where health information system is not present, 2) a difficulty arises when really large number of observations and pathways/outcomes are of interest. Although (generalized) linear mixed models are very flexible, computational issues arise with increasing number of observations and pathways. System Dynamics methodology is associated with some practical difficulties as building and validating SD models is a lengthy and arduous process, which requires teams with strong modelling skills and the availability of a large set of qualitative and quantitative data. The success of the process is heavily dependent on the engagement of the parties involved in the context of the study and this can burden these parties especially in the context of the UK health care where there are high demands on their time and attention.

The research focused on the modelling of a single unit and this may hide some of the important performance drivers as neonatal units are part of larger networks and this may hide the impact of network related factors such as admission policies and the availability of transportation resources between units. The research team is currently developing a new model at a network level as neonatal services are organised into networks in the UK. In addition, the performance indicators in this research focused primarily on the throughput from the neonatal units (number of babies exiting the unit per unit time). The evaluation of the performance would be more realistic if it included performance indicators related to the quality of care and its impact on the quality of life of the babies and investigated how these aspects may affect readmission of babies and the movement of babies between the different care states (IC, HDC, SC), which was found to be a main driver of the performance of neonatal units. 
The indicators used in this research to evaluate the performance of neonatal units did not include the indicators related to the quality of care and how care quality may have an impact on future readmissions of babies to the unit. These issues are been addressed in an extended model, which will include quality of care performance indicators. Furthermore, the new model represents a network of interconnected neonatal units and will explicitly portray the processes of admissions, readmissions, and transfer of babies between the different units in the network.

The methodologies used in this research offer decision makers powerful tools to appreciate the complexity of the neonatal system, understand its inner working, and the variables driving their behaviour and performance. In fact these methodologies are "tools for thinking" (Pidd, 2010) enabling managers to challenge their assumptions and see the systems in which they operate in a new light. Furthermore, they offer decision makers the opportunity to evaluate the implications of possible policies and actions on the performance of their systems before the actions are implemented in the real world, hence avoiding the trap of "doing things and hoping for the best". As such, policies are designed based on the solid ground offered by the formality and the analytical robustness of these methodologies, hence an evidence based decision making process.

To conclude, we can say that the current study has led to some important insights into the relationship between LoS and performance of neonatal systems. However, it has only added to our thirst to learn more about this important element of the UK health care provision system. The results of this research have already paved the road to the investigation of more issues regarding the management of neonatal services in the UK. For example, it would be very interesting to know the effects of resources allocation and management policies, changes to admission policies, integration of services, and restructuring of neonatal delivery structures on the performance of these systems.

The tough economic conditions are expected to be with us for quite a while in the future. Against this background and given the ever increasing demands for health care provision, the growing variety of health technologies, procedures, and health care delivery modes, and the increasing size and complexity of health systems structures, it is clear that a paradigm shift is required in the decision making and policy design processes in order to deal with this new reality. Modelling techniques 
are extremely well positioned to play a significantly greater role in shaping and influencing these processes and policies and to enable the UK health care system to face up to these challenges and improve the quality and performance of health care delivery and provision.

\section{References}

Aalen OO (1995). Phase type distributions in survival analysis. Scandinavian Journal of Statistics 22: 447-463.

Adeyemi S, Chaussalet TJ, Xie H and Millard PH (2007). Patients flow: a mixed-effects modelling approach to predicting discharge probabilities. Proceedings of the 20th IEEE International Symposium on Computer-Based Medical Systems. IEEE CBMS 2007, Maribor, Slovenia, 20-22 June 2007. IEEE, Los Alamitos, USA, pp. 725-730. ISBN 0769529054.

Adeyemi S and Chaussalet TJ (2009a). Models for extracting information on patient pathways. In: McClean, S. and Millard, P. and El-Darzi, E. and Nugent, C.D., (eds.) Intelligent patient management. Studies in Computational Intelligence (189). Springer, pp. 171-182. ISBN 9783642001789

Adeyemi S, Demir E and Chaussalet T J (2009b). The analyses of individual patient pathways: Investigating regional rariation in COPD readmissions. The XIII International Conference on Applied Stochastic Models and Data Analysis (ASMDA-2009). Vilnius, pp. 316-319.

Adeyemi S, Chaussalet TJ, Xie H and Asaduzaman Md (2010). Random effects models for operational patient pathways. Journal of Applied Statistics 37: 691-701.

Adeyemi S, Chaussalet TJ and Demir E (2011). Nonproportional random effects modelling of a neonatal unit operational patient pathways. Statistical Methods and Applications. DOI 10.1007/s10260-011-0174-z.

Asaduzzaman Md, Chaussalet TJ and Robertson NJ (2009). A loss network model with overflow for capacity planning in a neonatal unit. Annals of Operations Research 178:, 67-76. 
Atun RA, Lebcir MR, McKee M, Habicht J and Coker RJ (2007).Impact of joined-up HIV harm reduction and multidrug resistant tuberculosis control programmes in Estonia: system dynamics simulation model. Health Policy 81: 207-217.

Barlas Y. Formal aspects of model validity and validation in system (1996). System Dynamics Review 12: 183-210.

BLISS (2007). Special delivery or second class: are we failing special care babies in the UK? The Premature Baby Charity. http://www.bliss.org.uk/pdfs/babyreport_updateweb.pdf

Brailsford SC, Lattimer VA, Turnaras P and Turnbull JC (2004). Emergency and on demand health care: modelling a large complex system. Journal of the Operational Research Society 55: 34-42.

Cooper K, Brailsford S C, and Davies R (2007). Choice of modeling technique for evaluating health care interventions. Journal of the Operational Research Society 58:, 169-176.

Cote M (2000). Understanding patients flow. Production and Operations Management 28:, 8-10.

Cote M and Stein W (2007). A stochastic model for a visit to the doctor's office. Mathematical and Computer Modelling 45:, 309-323.

Dangerfield BC (1999). System dynamics applications to European health care issues. Journal of the Operational Research Society 50: 345-353.

Department of Health (2009). Toolkit for high quality neonatal services. Department of Health, London, UK.

Faddy MJ (1994). Examples of fitting structured phase-type distributions. Applied Stochastic Models and Data Analysis 10: 247-255.

Faddy MJ (1995). Phase-type distributions for failure times. Mathematical and Computer Modelling 22: 63-70.

Faddy MJ and McClean SI (1999). Analysing data on lengths of stay of hospital patients using phase type distributions. Applied Stochastic Models in Business and Industry 15: 311-317. 
Frick U, Rehm J, Krischke A and Cordingg C (1996). Length of stay in German psychiatric hospital as a function of patient and organizational characteristics-a multilevel analysis. International Journal of Methods in Psychiatric Research 8: 146-161.

Gunal MM and Pidd M (2010). Discrete event simulation for performance modelling in health care: A review of the literature. Journal of Simulation 4:, 42-51.

Irvine V and McClean SI (1994). Stochastic models for geriatric in-patient behaviour. IMA Journal of Mathematics Applied in Medicine 8 Biology 11: 207-216.

Jun JB, Jacobson SH and Swisher JH (1999). Application of discrete event simulation in health care clinics: A survey. Journal of the Operational Research Society 50:, 109-123.

Lane DC, Monefeldt C and Rosenhead JV (2000). Looking in the wrong place for healthcare improvements: A System dynamics study of an accident and emergency department. Journal of the Operational Research Society 51: 518-531.

Lebcir MR, Atun AR and Coker JR (2010). System Dynamic Simulation of Treatment Policies to Address Colliding Epidemics of Tuberculosis, Drug Resistant Tuberculosis, and injecting drug users driven HIV in Russia. Journal of the Operational Research Society 51: 518-531.

Lee AH, Ng AS and Yau KK (2001). Determinants of maternity length of stay: a gamma mixture risk-adjusted model. Health Care Management Science 4: 249-255.

McClean S and Millard PH (1993). Patterns of legth of stay after admission in geriatric medicine: an event history approach. The Statistician 42: 263-274.

National Audit Office (2008). Caring for Vulnerable Babies: The reorganisation of neonatal services in England. HC-101 Session 2007-2008.

Neonatal Data Analysis Unit 2010 Report: Care days and Reason for admission of term babies. http://www1.imperial.ac.uk/medicine/research/researchthemes/reprodscience/ paediatrics/neonatalmedicine/ndau/, Imperial College London, UK, accessed 20 December 2011. 
Pidd M. Tools for Thinking. Modelling in Management Science (2010). Wiley, Chichester, UK.

Proudlove N C, Black S, and Fletcher A (2007). OR and the challenge to improve the NHS: modeling for insight and improvement in in-patient flows. Journal of the Operational Research Society 58:, $145-158$.

R Development Core Team (2005). R: A language and environment for statistical computing, $\mathrm{R}$ Foundation for Statistical Computing, Vienna, Austria.

Ross S (2003). Introduction to probability models. Academic Press - Elsevier Science.

Sterman JD (2000). Business dynamics. Systems thinking and modeling for a complex world. McGraw Hill: Singapore.

Taylor K and Dangerfield BC (2005). Modelling the feedback effects of reconfiguring health services. Journal of the Operational Research Society 56: 659-675.

Thomas WH (1968). A model for predicting recovery progress in coronary patients. Health Services Research 3:, 185-213.

Townshend JP and Turner HS (2000). Analysing the effectiveness of Chlamydia screenings. Journal of the Operational Research Society 51: 812-824.

Tucker J (2002). Patient volume, staffing, and workload in relation to risk-adjusted outcomes in a random stratified sample of UK neonatal intensive care units: a prospective evaluation. Lancet 359:, 99-107.

Yau K, Lee A and Ng A (2003). Finite mixture regression model with random effects: Application to neonatal hospital length of stay. Computational Statistics and Data Analysis 41: 359-366.

Wolstenholme E (1999). Patient flow perspective of U.K. health services: Exploring the case for new "intermediate care". System Dynamics Review 15: 253-271. 